\title{
ESTUDIO DE LAS CONFIGURACIONES DE PUNTOS DE CONTROL TERRESTRE PARA FOTOGRAMETRÍA CON DRONE.
}

\author{
STUDY OF THE CONFIGURATIONS OF GROUND CONTROL POINTS FOR \\ PHOTOGRAMMETRY WITH DRONE.
}

SEBASTIÁN CISNEROS ${ }^{1}$; ÉRIKA GARCÍA ${ }^{1}$; KAREN MONTOYA ${ }^{1}$; IZAR SINDE ${ }^{1,2}$

${ }^{1}$ DEPARTAMENTO DE CIENCIAS DE LA TIERRA Y CONSTRUCCIÓN, UNIVERSIDAD DE LAS FUERZAS ARMADAS "ESPE". Avenida General Rumiñahui $\mathrm{s} / \mathrm{n}$ Sangolquí, Ecuador, iisinde@espe.edu.ec; smcisneros2@espe.edu.ec; eegarcía3@espe.edu.ec; kjmontoya@espe.edu.ec.

${ }^{2}$ DEPARTAMENTO DE INGENIERÍA AGROFORESTAL, UNIVERSIDAD DE SANTIAGO DE COMPOSTELA. Avenida Benigno Ledo s/n Lugo, España, izar.sinde@ rai.usc.es

Recibido: 13 de mayo de 2019 / Aceptado: 18 de junio de 2019

\section{RESUMEN}

Una de las tendencias actuales de la fotogrametría aérea es el uso de UAV (Unmanned Aerial Vehicle) o vehículos aéreos no tripulados. La toma de imágenes desde estos dispositivos es de gran ayuda en un sin número de aplicaciones, principalmente en el área de las ciencias geográficas y agropecuarias. Algunos de los productos más populares a nivel de investigación son los modelos digitales de superficies, las curvas de nivel, la cartografía, los ortomosaicos y las imágenes multiespectrales. Para lograr obtener productos que estén dentro de la calidad exigida por las normas, es necesario que el vuelo realizado tenga una relación directa con el terreno, es decir, las imágenes obtenidas deben estar georreferenciadas. El presente estudio propone obtener la mejor configuración de puntos de control teniendo en cuenta la cantidad y su distribución sobre el terreno. El primer paso fue la elaboración del diseño de una malla regular de puntos sobre la zona de estudio. Estos puntos fueron materializados en el terreno a través de un replanteo mediante topografía clásica utilizando una estación total y a continuación se obtuvieron coordenadas precisas a través de tecnología GNSS. El posicionamiento se realizó por el método fast static con equipos de una frecuencia y para finalizar se realizó el debido postproceso. Por último, se realizó el vuelo con drone de la zona para la obtención de imágenes con las que se realizaron los ortomosaicos para un total de 45 configuraciones diferentes de 3, 4, 5 y 6 puntos de control. Como se ha visto, para la fotogrametría con drone no existe una metodología que indique cuantos puntos y donde deben ser colocados ya que estos parámetros varían dependiendo del terreno en donde se va a realizar el proyecto, es por ello que más bien estos criterios están basados en la experiencia del operador. De esta forma, se pretende ofrecer una guía a la hora de seleccionar los puntos de control, para futuros trabajos de características muy similares al presente proyecto. La configuración que obtuvo menor error medio cuadrático (RMSE) fue una de las configuraciones de 5 puntos.

Palabras clave: UAV, GNSS, configuración de puntos, RMSE.

\begin{abstract}
One of the current trends in aerial photogrammetry and is the use of UAV (Unmanned Aerial Vehicle). The taking of images are of great help in a number of applications, mainly in the area of geographical and agricultural sciences. Some of the most popular products that this technology can generate are surface models, contour lines, cartography, ortomosaics and multispectral images. In order to get the best quality in the product, it is necessary that the performed flight had a direct relationship with the terrain, that is, the
\end{abstract}


images obtained must be dereferenced. In this study proposes to obtain the best control point configuration taking into account their amount and position on the ground. First it was done the design of a regular mesh of points on the study area. These points were defined in ground by stake out using total station. To obtain precise coordinates of the materialized points, GNSS technology was applied with fast static positioning. It was made with one frequency equipment and subsequently performed due post processing. Finally the drone flight was carried out in the area to obtain images that they were used to adjust the orthomosaics with the 45 configurations of $3,4,5$ and 6 points. As we have seen, for photogrammetry with drone there is no a standard methodology that indicates how many points and where they should be placed, rather the experience of the user is the main criterion to choose the control points. In this way, it is intended to offer a guide for future work with characteristics very similar to the present project. The configuration that obtained the lowest RMSE was the configuration of 5 points.

Keywords: UAV, GNSS, configuration of points, RMSE.

\section{INTRODUCCIÓN}

La fotogrametría aérea ha incrementado su desarrollo tecnológico y ha evolucionado a través del tiempo, es la encargada de calcular las dimensiones y posiciones de los objetos en el espacio, a partir de medidas realizadas en fotografías (Santos \& Albert, 2014). Estas fotografías son obtenidas desde vehículos aéreos (Otero, Ezquerra, Rodriguez-Solano, Martín, \& Bachiller, s.f.).

En la actualidad, el uso de vehículos aéreos no tripulados (U.A.V.) se ha incrementado enormemente. Un UAV se define como un vehículo aéreo motorizado que no lleva a bordo un operador humano, utiliza fuentes aerodinámicas para generar la sustentación y puede volar autónomamente o ser tripulado de forma remota (Claros, Guevara, \& Pacas, 2016).

Para lograr obtener resultados aceptables de los productos de un UAV respecto a las ciencias geográficas, es necesario que el vuelo realizado tenga una relación directa con el terreno, es decir, las imágenes obtenidas deben estar georreferenciadas mediante el uso de puntos de control terrestre. Se define a un punto de control como un sitio físico en la tierra del cual se conoce su verdadera posición respecto a un sistema de coordenadas y se puede utilizar como guía (Perdomo, et al., 2015).

Para establecer puntos de control terrestre en fotogrametría con drone, se usan diferentes metodologías según la conveniencia del profesional y las características topográficas del terreno o área de estudio, debido a que no existe una norma ya establecida que permita conocer cuál es la cantidad óptima de puntos de control terrestre.

Según Rivera (2016), aplicar 6 puntos de control para un área de 30 hectáreas, es suficiente, mientras que según Perdomo et al; (2015), establece un total de 12 puntos de control en un área de 6 hectáreas. 
Por otro lado, PhotoScan (2018), recomienda colocar de 10 a 15 puntos de control bien distribuidos en el área de interés, para lograr resultados de la más alta calidad.

La determinación de los puntos de control terrestre o puntos de apoyo pueden realizarse utilizando procedimientos clásicos de topografía o bien mediante la utilización de GNSS (Perez, 2001). El GNSS, Global Navigation Satellite System, es un conjunto de sistemas de navegación por satélite, como GPS, GLONASS, GALILEO, QZSS, IRNSS, Beidou. Es decir, aquellos sistemas que son capaces de dotar en cualquier punto y cualquier momento de posicionamiento espacial y temporal (García, 2008). Existen varios métodos de posicionamiento que se pueden aplicar con GNSS, su elección depende principalmente de la precisión requerida para el trabajo, del equipo que se vaya a utilizar y de la técnica de procesamiento de las observaciones (Garrido, 2016).

En la actualidad el apoyo de campo se realiza con receptores GNSS. En esencia el método GNSS de trabajo más utilizado es el estático-relativo que consiste en estacionar un receptor fijo en un punto de coordenadas conocidas, como por ejemplo un vértice perteneciente a una determinada red geodésica y otro receptor que se va movilizando por todos los puntos de apoyo de los cuales es necesario conocer sus coordenadas (Organización de Aviación Civil Internacional, 2011).

En el trabajo de Prajwa, Rishab, Vaibhav, \& Karthik (2016) se utiliza el método GNSS diferencial para la recolección de un total de nueve puntos de control terrestre a lo largo de un terreno de 650 metros cuadrados, con el objetivo de evaluar cual es la cantidad óptima de puntos de control en tierra para mantener la precisión en el área de estudio utilizando un UAV, obteniendo que para 600 metros se necesitan 3 puntos de control.

En Forlani, et al. (2018) se utilizaron receptores geodésicos doble frecuencia y diferentes técnicas GNSS como el método Stop and Go, y RTK para obtener las coordenadas de 11 Ground Control Points (GCP's) utilizados en el procesamiento de las imágenes. En cambio, en Gonçalves, Pérez \& Duarte (2018) en un terreno de aproximadamente 400 hectáreas, para la adquisición de los puntos de control terrestre se utilizó el método GNSS Stop and Go y concluyendo que la cantidad óptima de GCP's varían entre 18 y 24 puntos y deben ser distribuidos homogéneamente sobre la superficie del terreno.

Al no existir una determinada metodología, que indique la cantidad y configuración adecuada de puntos de control terrestre que se necesitan para la fotogrametría con drone, se planteó este estudio con el objetivo de conocer cuál es la cantidad y configuración más adecuada de puntos de control terrestre que permitan obtener productos derivados de imágenes de drone que tengan precisiones aceptables según la norma vigente en el Ecuador. 


\section{1.ÁREA DE ESTUDIO}

El área de estudio está ubicada en la provincia de Pichincha, cantón Rumiñahui, a 40 Km de la ciudad de Quito, en el campus politécnico Hacienda El Prado IASA I. Este campus se encuentra en el piso altitudinal montano bajo. En la figura 1 se indica en color rojo el área establecida dentro del campus para el cultivo de pasto donde se centrará el trabajo del presente proyecto, con una superficie de $8335\left[\mathrm{~m}^{2}\right]$. Cabe mencionar que los linderos del terreno, son de árboles de eucalipto, por lo tanto, al ser árboles de una altura considerable, tienen influencia en la toma de los datos GNSS.

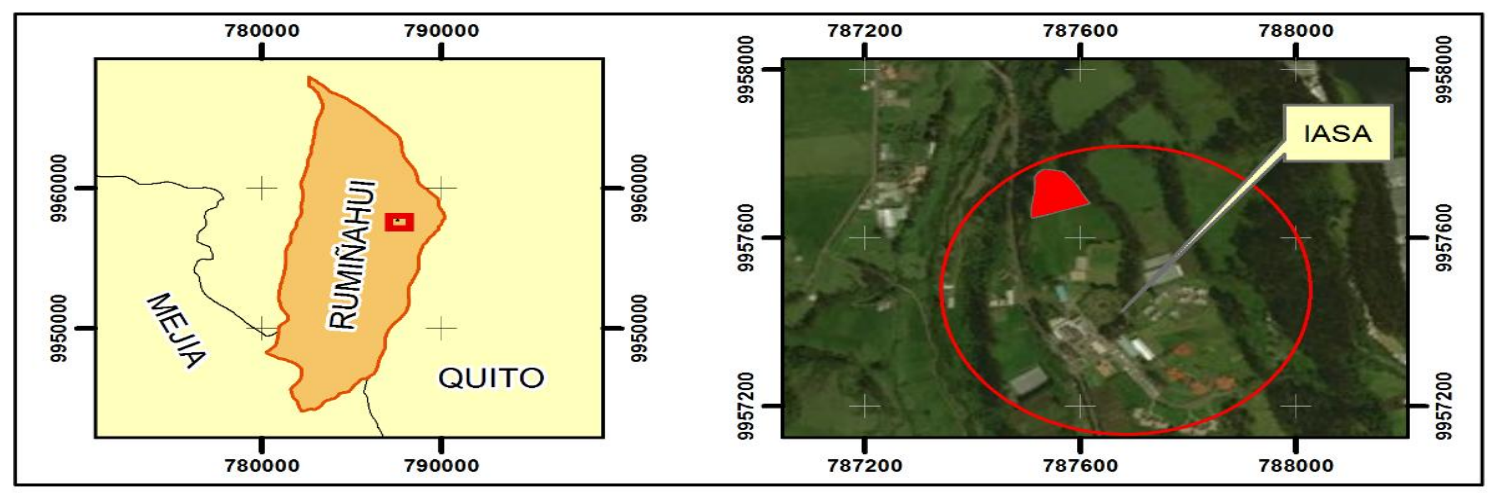

Figura 1. Localización del área de estudio.

\section{METODOLOGÍA}

En la figura 2 se muestra el flujograma de la metodología empleada en esta investigación.

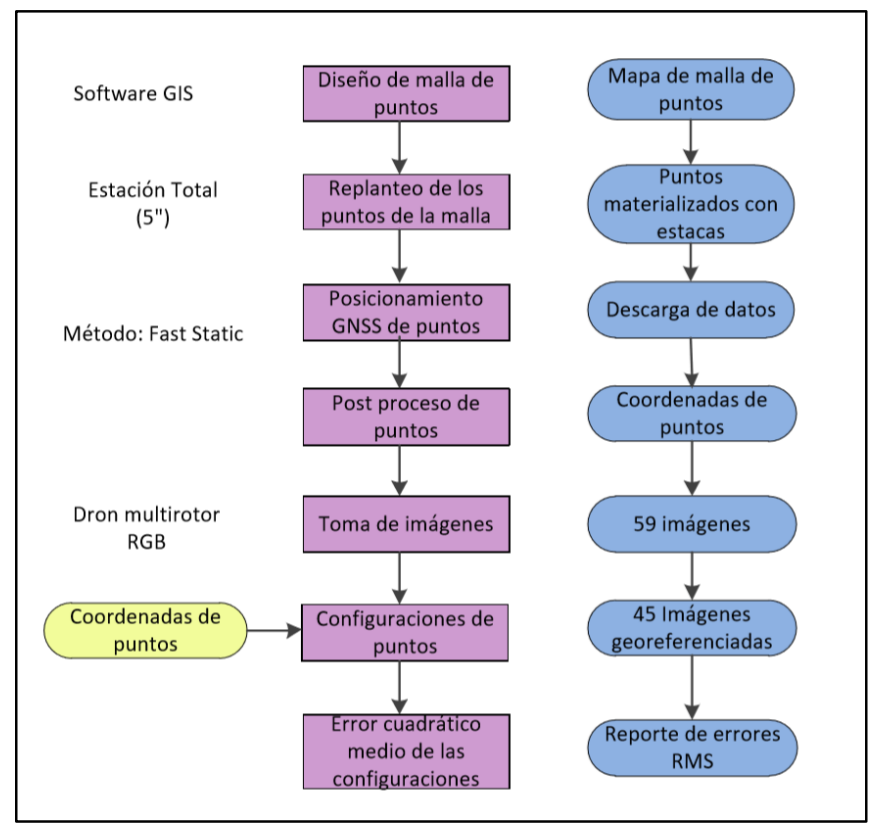

Figura 2. Modelo cartográfico de la metodología aplicada. 


\subsection{DISEÑO Y REPLANTEO DE LA MALLA DE PUNTOS}

De acuerdo al área del terreno se realizó una malla de puntos, utilizando un software GIS. Se procedió a ubicar los puntos de control cada 20 metros en sentido sur - norte y cada 20 metros en sentido este - oeste, mediante el método de replanteo con una estación total de 5" de precisión. De esta forma se obtuvieron 18 puntos, materializados mediante estacas dentro del área de estudio. Posteriormente estos puntos replanteados, fueron posicionados con GNSS de precisión como se expone en el apartado 2.2.

\subsection{POSICIONAMIENTO GNSS DE PUNTOS DE CONTROL}

Para la toma de los puntos, se aplicó la técnica de posicionamiento estático rápido (fast static), que se llevó a cabo con los equipos de doble frecuencia (Precisión Horizontal: $3 \mathrm{~mm}+0.5 \mathrm{ppm}$ RMS, Precisión Vertical 5mm +0.5ppm RMS) como base y 2 móviles de una frecuencia. Se ubicaron los dos móviles durante 10 minutos en cada punto, con un intervalo de registro de 5".

Como primer paso para ejecutar este método se deben obtener las coordenadas de un punto conocido que actúa de base y que a la vez se encuentre en las proximidades del lugar donde se quiere hacer el levantamiento. Se utilizó como punto base el punto 5 del estudio de Grefa \& Sánchez (2018), punto que fue rastreado mediante el método estático rápido empleando como base para su posicionamiento la estación EPEC, perteneciente a la infraestructura de monitoreo continuo del Ecuador (REGME) y cuyas coordenadas en el sistema de referencia WGS-84 UTM 17 Sur son (787549.228; 9957716.160; 2706.566). Cuanto más próxima se encuentre la citada base, se generará un vector más corto, lo que facilita que existan menos errores. En general es recomendada una línea base que no exceda los 14 kilómetros (Ayala \& Hasbun, 2012). En este caso el equipo cuenta con un error de $3 \mathrm{~mm}+0.5 \mathrm{ppm}$ RMSE, lo que genera precisiones de hasta $1 \mathrm{~cm}$, con distancia máxima hasta la base de $10 \mathrm{~km}$, utilizando equipos de una frecuencia.

En este punto es importante mencionar que, de los 18 puntos de la malla inicial, 4 de ellos no entraron en la precisión de $10 \mathrm{~cm}$, por lo que se decidió no tenerlos en cuenta en el estudio. Este error en su posicionamiento puede ser debido a la interferencia de los árboles de eucalipto que se ubicaban cerca de estos puntos.

\subsection{PLAN DE VUELO Y TOMA DE IMÁGENES CON DRONE.}

Para el plan de vuelo con drone, se utilizó un software de procesamiento de imágenes de drone, en la Tabla 1 se detallan los parámetros del plan de vuelo. Se realizó la toma de 59 imágenes utilizando un drone multirotor con cámara RGB de 12 megapíxeles. 
Tabla 1. Parámetros del plan de vuelo.

\begin{tabular}{|c|c|}
\hline Altura de vuelo & 70 metros \\
\hline Número de líneas & 6 con sentido al noroeste \\
\hline Traslapo longitudinal & $80 \%$ \\
\hline Traslapo transversal & $70 \%$ \\
\hline Resolución espacial & $3 \mathrm{~cm}$ por píxel \\
\hline Tiempo de vuelo & 5 minutos 11 segundos \\
\hline
\end{tabular}

\subsection{CONFIGURACIONES DE LOS PUNTOS}

Se establecieron 15 configuraciones con 3 puntos de control y 10 configuraciones con 4 , 5 y 6 puntos de control. Además del número de puntos, se tuvo en cuenta su configuración en función de si son puntos extremos, puntos internos o una combinación de los dos. Para evitar el posible sesgo por carencias visuales o pericia del operador, se realizó el pinchado de los puntos de control (GCP`s) por diferentes personas, para las distintas configuraciones propuestas. Para la generación de las 45 ortoimágenes se utilizó un software de procesamiento de imágenes de drone de tipo comercial. La precisión de las ortoimágenes varían en función de las distintas configuraciones de puntos de control planteadas en el paso anterior.

\subsection{ESTUDIO DE LA CONFIGURACIÓN DE LOS PUNTOS}

En un software GIS, se procedió a cargar las imágenes georreferenciadas, y los puntos de control de las distintas configuraciones, para medir la diferencia que existe entre ellas. Con este valor se determinó el error medio cuadrático de cada configuración para poder decidir cuál es la mejor para este terreno.

El error medio cuadrático es aquel cuyo cuadrado es igual a la media aritmética de los cuadrados de los errores verdaderos, definiéndose por la siguiente ecuación (FGDC, 1998):

$$
R M S E=\sqrt{\frac{\sum_{i=1}^{n}(\text { Cartografía-Control })^{2}}{n}}
$$

Donde:

- Cartografía representa los datos obtenidos en campo. 
- Control representa los datos de referencia

- $\mathrm{n}$ representa el número de puntos de verificación aprobados.

También se realizó el cálculo del Estándar Nacional para la Precisión de Datos Espaciales NSSDA (National Standard for Spatial Data Accuracy), que implementa una metodología estadística para estimar la precisión posicional de puntos en mapas y en datos geoespaciales digitales, respecto a posiciones terrestres georreferenciadas de mayor precisión (FGDC, 1998).

Analiza la componente horizontal (XY conjuntamente) y la componente vertical (Z) proyectadas en UTM 17 Sur, de puntos empleando fuentes de mayor exactitud. Con la obtención del RMSE para XY y para Z, calcula el error real de la muestra analizada en función de un determinado nivel de confianza impuesto por el usuario (generalmente $95 \%)$.

La precisión se informa en distancias terrestres con un nivel de confianza del 95\% lo que significa que el $95 \%$ de las posiciones en el conjunto de datos tendrá un error con respecto a la posición de terreno real que sea igual o menor al valor de precisión informado (FGDC, 1998). El NSSDA muestra un índice de calidad de la cartografía en unidades reales sobre el terreno (Atkinson, García, \& Ariza, 2001)

Se seleccionó una muestra de 14 puntos, con base en el estándar según (FGDC, 1998), pero lo que se recomienda es que se utilicen 20 puntos. Se calculó el RMSEx para la componente $X$ según la ecuación 1 y de igual manera para componente $Y$, posteriormente se calculó el RMSEr para la componente posicional $(X Y)$ según la ecuación 2 mencionada en FGDC (1998):

$$
R M S E_{r}=\sqrt{R M S E_{x}^{2}+R M S E_{y}^{2}}
$$

Como se indica en FGDC (1998) se calculó el coeficiente de exactitud posicional a un $95 \%$ de confianza dependiendo de los siguientes casos:

a) Si: RMSEx = RMSEy, empleando la ecuación 3:

$$
\text { Exactitud }=1.7308 \cdot R M S E_{r}
$$

b) Si: RMSEx $\neq$ RMSEy, empleando la ecuación 4:

$$
\text { Exactitud }=1.22385 \cdot\left(R M S E_{x}+R M S E_{y}\right)
$$


En la generación de productos fotogramétricos a partir de imágenes digitales de drone existen numerosos errores y valores atípicos. Como se menciona en Gonçalves, Pérez, \& Duarte (2018) estos errores pueden provenir de:

- La etapa de adquisición de datos;

- El método utilizado por el software para la comparación de imágenes;

- El método de interpolación utilizado para llenar los vacíos que se generan;

- La complejidad de la superficie del terreno, entre otros.

Para la correcta utilización del estadístico RMSE, los datos deben estar distribuidos normalmente y no deben presentar valores atípicos. En el presente trabajo se encontró que los datos no tienen una distribución normal y presentan varios valores atípicos, razón por la que se utilizó un estadístico de precisión robusto, el error medio cuadrático robusto RRMSE (Robust Root Mean Mean Square).

El RRMSE es un estadístico de evaluación de precisión robusta que no toma en cuenta la distribución de los datos ni los valores atípicos. Para estimar el valor del RRMSE, se toman las medidas robustas y la desviación media absoluta normalizada (NMAD) como valores representativos de la media y desviación estándar de los residuos y se calcula como se muestra en la ecuación 6 propuesta en Gonçalves, Pérez, \& Duarte (2018):

Dónde:

$$
R R M S E=\sqrt{N M A D^{2}+M e^{2}}
$$

- NMAD es la desviación media absoluta normalizada, estimador robusto que se calcula de acuerdo a la ecuación 7:

$$
N M A D=1.4826 \cdot \text { Mediana }(\mid \text { Datos }- \text { Me } \mid)
$$

- "Me" es la mediana de los datos.

En la Tabla 2 se pueden visualizar los resultados del RMSE, RRMSE y del NSSDA de las 45 configuraciones con 3, 4, 5 y 6 puntos de control obtenidos mediante la ecuación 1 y la ecuación 4. En la Tabla 3, se detalla las medias de RMSE, RRMSE Y NSSDA de las configuraciones con 3, 4, 5 y 6 puntos de control, mientras que en la Tabla 4, se detallan porcentajes del total de configuraciones. 


\section{RESULTADOS Y DISCUSIONES}

Tabla 2. Resultados de las 45 configuraciones.

RMSE, RRMSE Y NSSDA DE LAS CONFIGURACIONES CON 3, 4, 5 Y 6 PUNTOS DE CONTROL

\begin{tabular}{|c|c|c|c|c|c|}
\hline $\begin{array}{c}\text { No. de } \\
\text { Puntos de } \\
\text { Control }\end{array}$ & $\begin{array}{c}\text { No. } \\
\text { Configuración }\end{array}$ & $\begin{array}{c}\text { RMSE } \\
(\mathbf{c m})\end{array}$ & $\begin{array}{c}\text { RRMSE } \\
\text { (cm) }\end{array}$ & $\begin{array}{l}\text { NSSDARMSE } \\
\text { (cm) }\end{array}$ & $\begin{array}{l}\text { NSSDARRMSE } \\
(\mathrm{cm})\end{array}$ \\
\hline \multirow{15}{*}{ 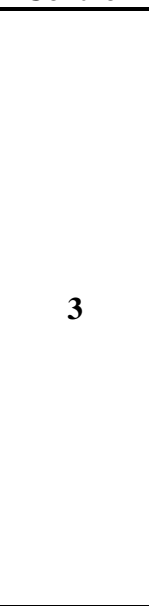 } & 1 & 26.3511 & 22.2344 & 32.2497 & 27.21154791 \\
\hline & 2 & 27.2587 & 23.2398 & 33.3606 & 28.44200925 \\
\hline & 3 & 27.9424 & 24.8980 & 34.1973 & 30.47141861 \\
\hline & 4 & 17.3250 & 16.3152 & 21.2032 & 19.96741348 \\
\hline & 5 & 28.2237 & 24.8085 & 34.5415 & 30.36182655 \\
\hline & 6 & 26.8744 & 24.0111 & 32.8902 & 29.38597426 \\
\hline & 7 & 28.4768 & 7.8323 & 34.8514 & 9.585571175 \\
\hline & 8 & 28.7170 & 11.9341 & 35.1453 & 14.60559515 \\
\hline & 9 & 30.1873 & 6.7013 & 36.9448 & 8.201422962 \\
\hline & 10 & 29.3965 & 10.6731 & 35.9770 & 13.06227574 \\
\hline & 11 & 29.7094 & 8.1725 & 36.3599 & 10.0019553 \\
\hline & 12 & 30.3819 & 10.7305 & 37.1829 & 13.1325445 \\
\hline & 13 & 29.4906 & 30.2948 & 36.0920 & 37.07625385 \\
\hline & 14 & 68.8090 & 73.2624 & 84.2119 & 89.66220768 \\
\hline & 15 & 31.3716 & 11.3338 & 38.3941 & 13.87083903 \\
\hline \multirow{10}{*}{4} & 1 & 27.7305 & 19.1678 & 33.9380 & 23.45850676 \\
\hline & 2 & 32.3230 & 9.3554 & 39.5585 & 11.44956317 \\
\hline & 3 & 39.2271 & 38.2990 & 48.0081 & 46.87221447 \\
\hline & 4 & 31.5978 & 8.1129 & 38.6709 & 9.928956258 \\
\hline & 5 & 31.3419 & 29.1320 & 38.3578 & 35.65315543 \\
\hline & 6 & 32.0179 & 29.7850 & 39.1851 & 36.45238996 \\
\hline & 7 & 30.7221 & 6.6931 & 37.5992 & 8.191355822 \\
\hline & 8 & 26.0666 & 15.2236 & 31.9016 & 18.63142676 \\
\hline & 9 & 29.2379 & 9.8788 & 35.7828 & 12.09019466 \\
\hline & 10 & 28.1512 & 19.2987 & 34.4528 & 23.6186553 \\
\hline \multirow{10}{*}{5} & 1 & 57.1837 & 52.5043 & 69.9843 & 64.25737554 \\
\hline & 2 & 27.0096 & 18.1233 & 33.0557 & 22.18020316 \\
\hline & 3 & 32.2104 & 5.6063 & 39.4208 & 6.861230068 \\
\hline & 4 & 6.2259 & 6.5354 & 7.6196 & 7.998330767 \\
\hline & 5 & 31.8503 & 9.9736 & 38.9800 & 12.20613413 \\
\hline & 6 & 31.3299 & 7.8281 & 38.3431 & 9.580435157 \\
\hline & 7 & 25.8331 & 18.6659 & 31.6158 & 22.8443012 \\
\hline & 8 & 26.6825 & 16.3608 & 32.6554 & 20.0232132 \\
\hline & 9 & 17.2260 & 6.3638 & 21.0821 & 7.788320039 \\
\hline & 10 & 32.4649 & 8.6993 & 39.7322 & 10.64658302 \\
\hline \multirow{10}{*}{6} & 1 & 26.8584 & 25.7696 & 32.8707 & 31.53807381 \\
\hline & 2 & 33.4847 & 5.7623 & 40.9803 & 7.052158261 \\
\hline & 3 & 27.3742 & 11.9687 & 33.5019 & 14.64793846 \\
\hline & 4 & 25.7723 & 24.0023 & 31.5414 & 29.37520777 \\
\hline & 5 & 18.0828 & 8.0999 & 22.1307 & 9.913037559 \\
\hline & 6 & 37.6444 & 40.1562 & 46.0711 & 49.14512971 \\
\hline & 7 & 28.1615 & 16.6654 & 34.4655 & 20.39591284 \\
\hline & 8 & 27.8127 & 16.3196 & 34.0385 & 19.97270977 \\
\hline & 9 & 27.4742 & 12.3390 & 33.6243 & 15.1010941 \\
\hline & 10 & 29.4686 & 5.4045 & 36.0652 & 6.614258056 \\
\hline
\end{tabular}


Tabla 3. Media de los errores.

\begin{tabular}{|c|c|c|c|c|}
\hline \multicolumn{5}{|c|}{ MEDIAS DE RMSE, RRMSE Y NSSDA DE LAS CONFIGURACIONES CON 3, 4, 5 Y 6} \\
PUNTOS DE CONTROL \\
\hline $\begin{array}{c}\text { No. de Puntos de } \\
\text { Control }\end{array}$ & RMSE (cm) & RRMSE (cm) & NSSDARMSE (cm) & NSSDARRMSE (cm) \\
\hline $\mathbf{3}$ & 30.7010 & 20.4295 & 37.5735 & 25.0026 \\
\hline $\mathbf{4}$ & 30.8416 & 18.4946 & 37.7455 & 22.6346 \\
\hline $\mathbf{5}$ & 28.8016 & 15.0661 & 35.2489 & 18.4386 \\
\hline $\mathbf{6}$ & 28.2134 & 16.6487 & 34.5290 & 20.3756 \\
\hline
\end{tabular}

Tabla 4. Porcentajes de aceptación.

\begin{tabular}{|c|c|c|c|c|}
\hline \multicolumn{5}{|c|}{ PORCENTAJES DEL TOTAL DE CONFIGURACIONES. } \\
\hline \multirow{2}{*}{ Estadístico } & Precisión de $30[\mathrm{~cm}]$ & \multicolumn{2}{c|}{ Precisión de $10[\mathrm{~cm}]$} \\
\cline { 2 - 5 } & Aceptable & Rechazo & Aceptable & Rechazo \\
\hline RMSE & $62 \%$ & $38 \%$ & $2 \%$ & $98 \%$ \\
\hline RRMSE & $89 \%$ & $11 \%$ & $36 \%$ & $64 \%$ \\
\hline NSSDARMSE & $9 \%$ & $91 \%$ & $2 \%$ & $98 \%$ \\
\hline NSSDARRMSE & $78 \%$ & $22 \%$ & $24 \%$ & $76 \%$ \\
\hline
\end{tabular}

Para la obtención de la mejor configuración y cantidad necesaria de puntos de control terrestre se realizaron distintas configuraciones a partir de los 14 puntos obtenidos con GPS; se realizaron 15 configuraciones con $3 \mathrm{GCP}, 10$ configuraciones con $4 \mathrm{GCP}$, con 5 GCP y con $6 \mathrm{GCP}$, es decir, se obtuvo un total de 45 diferentes configuraciones de puntos de control.

Debido a que el proyecto es diseñado para una escala 1: 1000, la precisión del producto final es de 30 [cm] (IGM, 2006). En forma estricta, los $30 \mathrm{~cm}$, es lo que exige la norma, pero como no es el producto final algunos autores como Sevilla (1991), recomiendan que la precisión sea un tercio de la precisión final.

Como resultados de las distintas configuraciones realizadas con 3 puntos de control, se observa que la configuración número 4 presenta el menor RMSE $(17.3[\mathrm{~cm}])$, y la peor configuración fue la número 14, presentando un RMSE de 68.8 [cm]. La configuración número 9 presenta el menor RRMSE $(6.7[\mathrm{~cm}])$ y de igual manera la configuración número 14 es la peor con un RRMSE de $73.3[\mathrm{~cm}]$.

De las 10 diferentes configuraciones con 4 puntos, se obtuvo que la mejor fue la octava configuración, con un RMSE igual a 26.1 [cm], la configuración número 7 presenta el menor RRMSE (6.7 [cm]), alcanzando la precisión requerida para el proyecto. 
El RMSE más bajo de todo el proyecto fue con la configuración cuarta de 5 puntos de control, dando como resultado un valor de 6.2 [cm] y el RRMSE más bajo fue el de la configuración 10 con seis puntos de control, obteniendo un valor de $5.4[\mathrm{~cm}]$.

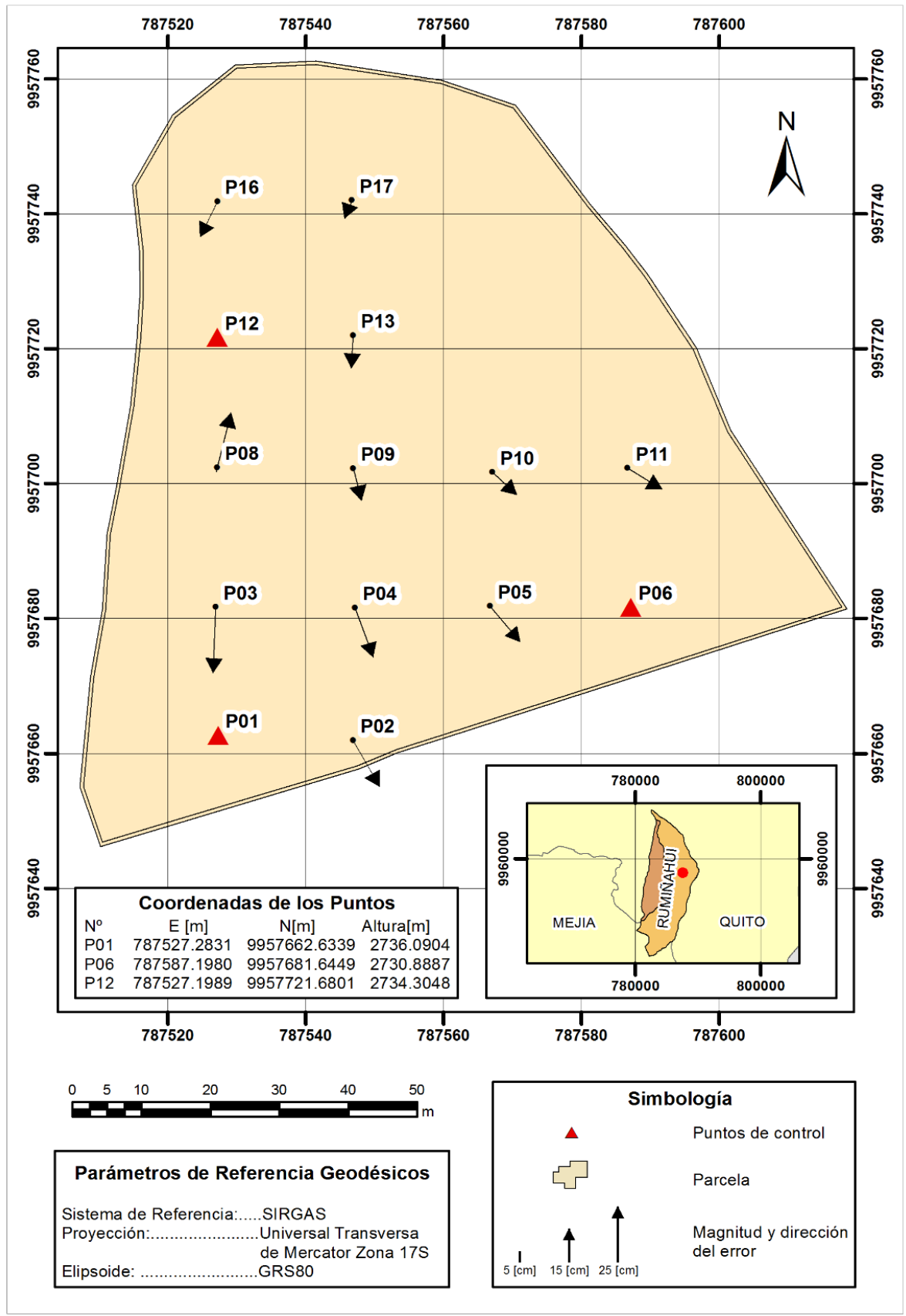

Figura 3. Mapa de localización de la menor cantidad de puntos de control que presentan el mayor RMSE ( 3 puntos) y direcciones de los errores. 


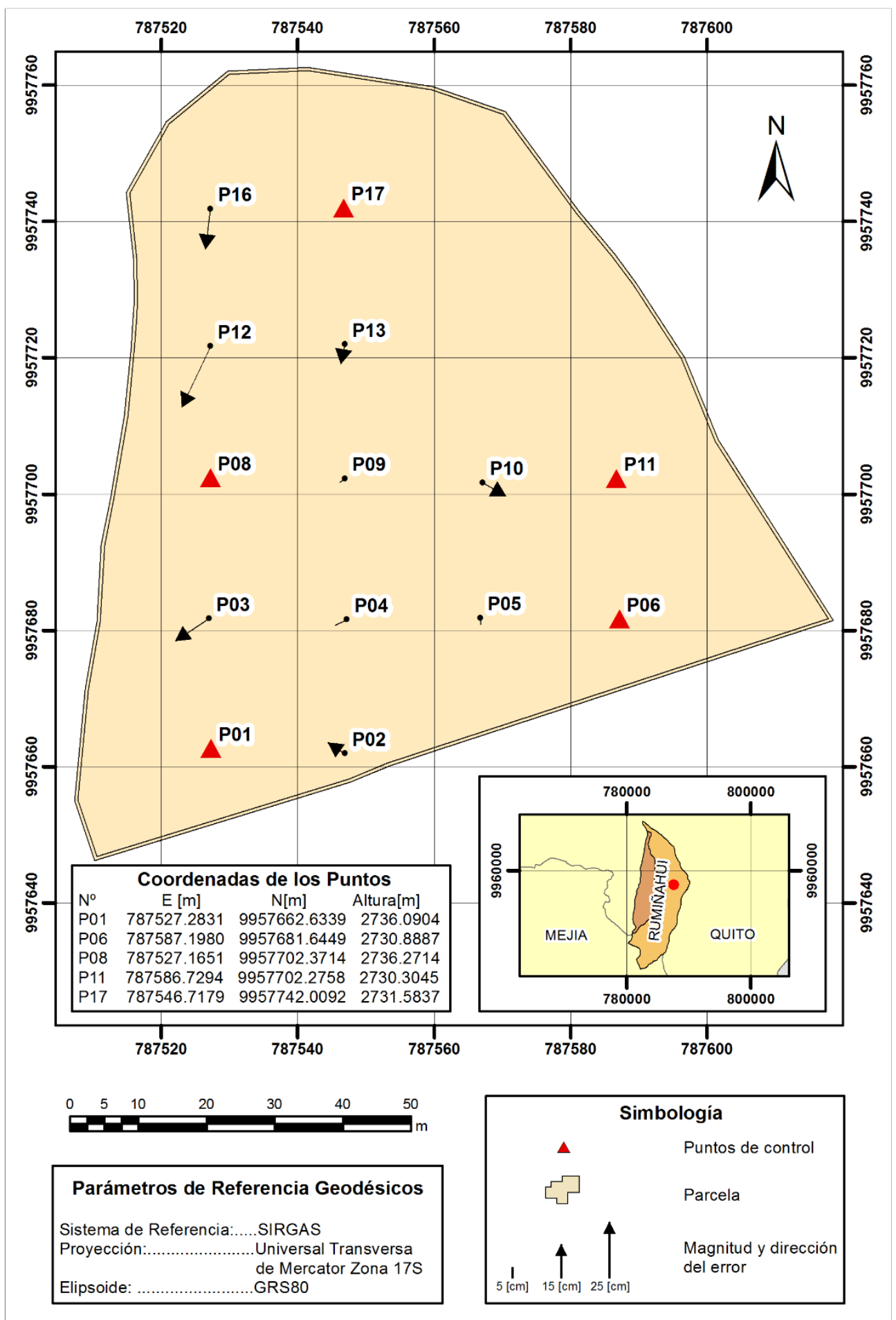

Figura 4. Mapa de localización de los puntos de control que presentan el menor RMSE (5 puntos) y direcciones de los errores. 


\section{CONCLUSIONES}

Los errores evidenciados en las figuras 3 y 4, ubicados en la parte izquierda del área de estudio, pueden ser provocados debido a la presencia de árboles de eucalipto alrededor del terreno.1

Como resultados de las distintas configuraciones realizadas con 3 puntos de control, es posible concluir que la $4^{\text {ta }}$ configuración obtuvo el menor RMSE con $17.3[\mathrm{~cm}]$, esto se debe a que la configuración toma en cuenta los puntos de los extremos de la parcela. Del total, 28 configuraciones superan la precisión de 1:1000 lo que representa el 62\% de casos exitosos.

De las 10 diferentes configuraciones con cuatro puntos, se obtuvieron 4 configuraciones $\left(1^{\text {era }}, 8^{\text {va }}, 9^{\text {na }}\right.$ y $\left.10^{\text {ma }}\right)$, el $40 \%$, con RMSE menor a la precisión del producto. De estas 4 configuraciones se pudo observar que la $8^{\text {va }}$ configuración obtuvo un RMSE igual a 26.1 [cm], lo que supone el menor RMSE dentro de las configuraciones de 4 puntos.

Con las configuraciones realizadas con 5 puntos de control, se obtuvieron 6 configuraciones que cumplen con la precisión requerida $\left(2^{\mathrm{da}}, 4^{\text {ta }}, 6^{\text {ta }}, 7^{\text {ma }}, 8^{\mathrm{va}}\right.$ y $\left.9^{\text {na }}\right)$, el $60 \%$. Siendo la $4^{\text {ta }}$ configuración la mejor, con un RMSE igual a $6.2259[\mathrm{~cm}]$.

Se obtuvo 8 configuraciones $(80 \%)$ que se encuentran dentro de la precisión requerida utilizando 6 puntos de control, siendo la configuración número 5 la de menor RMSE, con un RMSE igual a 18.0828 [cm].

La $4^{\text {ta }}$ configuración realizada con 6 puntos de control es la que menor error cuadrático medio presenta, en comparación a todas las configuraciones realizadas, esto debido a que se tiene mayor cantidad de puntos. En cambio, la configuración que presenta la menor cantidad de puntos de control con unos resultados aceptables según la norma, es la $4^{\text {ta }}$ configuración de 3 puntos de control.

La configuración con mayor RMSE (68.8090) [cm] fue la configuración $14^{\text {va }}$ realizada con 3 puntos de control y esto se debe a que los puntos de control están concentrados motivo por el que aumentan los errores en las zonas próximas a los extremos.

El RRMSE, al tratarse de un estimador más robusto, evidencia que los resultados son más permisivos que los otros dos estimadores, superando la tolerancia establecida en prácticamente todos los casos.

El NSSDA, al ser un estadístico estandarizado debe necesariamente calcularse con los valores de RMSE, sin embargo, los valores varían si se calculan con el RRMSE, resultando menores que los originales. 
Las configuraciones que cumplen con la precisión requerida son las que tienen puntos de control en los lugares extremos.

La probabilidad de realizar un ajuste correcto aumenta un $20 \%$ por cada punto de control que se añade.

Del total de configuraciones, el $62 \%$ es aceptable para la precisión de 30 [cm] de RMSE que exige el IGM y si se considera el RRMSE, el $89 \%$ es aceptable. En cambio para la el criterio de $1 / 3$ de la precisión del producto final, es decir 10 [cm], tan solo el $2 \%$ fue aceptable para el RMSE y el 36\% para el RRMSE.

La configuración de los puntos es de vital importancia cuando se tiene un número de puntos limitados. Tan solo las que tienen una distribución uniforme y desfocalizada, alcanzan una buena precisión. Este criterio se diluye en el momento que se añaden más puntos, ya que se evidencia que las probabilidades de obtener un producto aceptable aumentan. En este sentido es importante encontrar un balance entre el número de puntos de control y la superficie en estudio, de forma que el apoyo terrestre no encarezca el proyecto de forma innecesaria.

Este estudio se enfoca a una superficie de pequeñas dimensiones, acorde a las necesidades de un estudio focalizado de agricultura de precisión no obstante, se recomienda en el futuro realizar un trabajo que abarque una mayor superficie, con diferentes factores topográficos y un número mayor de puntos de control.

\section{REFERENCIAS}

Atkinson, A., García, J., \& Ariza, F. (2001). Los Diferentes Test Para El Control De Calidad Posicional En Cartografía.

Ayala, A., \& Hasbun, M. (2012). Aplicaciones Y Uso De La Tecnología De GPS Diferencial De Doble Frecuencia Con Precisión Centimétrica En El Área De Levantamiento Y Replanteo Topográfico Georeferenciado. Ciudad Universitaria: UNIVERSIDAD DE EL SALVADOR.

Claros, R., Guevara, A., \& Pacas, N. (Agosto de 2016). Aplicación De Fotogrametría Aérea En Levantamientos Topográficos Mediante El Uso De Vehículos Aéreos No Tripulados. Obtenido de Universidad de El Salvador: http://ri.ues.edu.sv/14218/1/50108282.pdf

FGDC. (1998). Geospatial Positioning Accuracy Standards Part 3: National Standard for Spatial Data Accuracy.

Forlani, G., Dall'Asta, E., Diotri, F., Morra di Cella, U., Riccardo, R., \& Santise, M. (2018). Quality Assessment of DSMs Produced from UAV Flights Georeferenced with On-Board RTK Positioning.

Galván, J., Goncalves, G. R., \& Pérez, J. A. (2018). The impact of number and spatial distribution of GCPs on the positional accuracy of geospatial products derivedfrom low cost UASs.

Galván, J., Goncalves, G. R., \& Pérez, J. A. (s.f.). jshgdauegrfył wie. 
García,

D.

(2008).

Sistema

GNSS

(http://arantxa.ii.uam.es/ jms/pfcsteleco/lecturas/20080125DavidGarcia.pdf ed.).

Garrido, N. (2016). Métodos de posicionamiento GNSS GPS. Clasificación. Obtenido de https://nagarvil.webs.upv.es/metodos-de-posicionamiento-gnss-gps/

Gonçalves, G. R., Pérez, J. A., \& Duarte, J. (2018). Accuracy and effectiveness of low cost UASs and open source photogrammetric software for foredunes mapping. Portugal: International Journal of Remote Sensing.

Grefa, J. \& Yánez, D., 2018. Análisis de la correlación entre el volumen vegetal de pasto con las características del suelo mediante técnicas de UAV, Quito: Universidad de las Fuerzas Armadas ESPE.

IGM. (2006). Ley de Cartografía.

López, F., López, C., \& Pérez, C. (2006). Elementos de topografía y construcción (Tercera Edición ed.). Oviedo: Ediciones de la Universidad de Oviedo.

Organización de Aviación Civil Internacional. (2011). Sistemas de aeronaves. Montréal, Quebec, Canada: ICAO.

Otero, I., Ezquerra, A., Rodriguez-Solano, R., Martín, L., \& Bachiller, I. (s.f.). Fotogrametría. Obtenido de http://ocw.upm.es/.../fotogrametria_cap_libro.pdf

Perdomo, C., Caicedo, J., Noldin, N., Machado, D., García, J., \& Pineda, M. (2015). Establecimiento de puntos de control terrestre para la corrección planialtimetrica de imágenes tomadas por drones. Venezuela.

Perdomo, C., Caicedo, J., Nuñez, N., Machado, D., García, J., \& Corina, M. (2015). Establecimiento de puntos de control terrestre para la corrección planialtimétrica de imágenes tomadas por drones. ResearchGate.

Perez, J. (2001). Apuntes de Fotogrametría - Centro Universitario de Mérida . Obtenido de http://redgeomatica.rediris.es/cartoprofesores/Fotogrametria/ApuntesFotogrametria3.pdf

PhotoScan, A. (2018). Agisoft PhotoScan User Manual: Professional Edition, Version 1.4. Agisoft LLC.

Prajwal, M., Rishab, J., Vaibhav, S., \& Karthik, K. (2016). Optimal Number of Ground Control Points for a UAV based Corridor Mapping.

Rivera, J. (2016). Evaluación de Método de Corrección Geométrica de Fotografía Aérea escala 1:1.000 capturada por vehículos aéreos no tripulados estableciendo una fred Geodésica de cuarto orden. Cuenca: Universidad del Azuay.

Santos, D., \& Albert, F. (2014). Fotogrametría usando plataforma aérea UAV(Unmanned Aerial Vehicle). Obtenido de Universidad Politécnica de Catalunya: https://core.ac.uk/download/pdf/41806759.pdf

Sevilla, M. (1991). Criterios de precisión cartográfica. Catastro, Año III, Num. 8. Instituto de Astronomía y Geodesia. Universidad Complutense de Madrid, Madrid, España, p.p 1220.

Trimble. (2014). USER GUIDE GNSS Receiver Module. Sunnyvale: Trimble. 